\title{
Probing CENP-E function in chromosome dynamics using small molecule inhibitor syntelin
}

\author{
Cell Research (2010) 20:1386-1389. doi:10.1038/cr.2010.167; published online 30 November 2010
}

\section{Dear Editor,}

Chromosome movements during mitosis are orchestrated primarily by the interaction of spindle microtubules with the kinetochore [1], the site for attachment of spindle microtubules to the centromere. The kinetochore has an active function in chromosomal segregation through microtubule-based motors located at or near it [1-2]. CENP-E is a microtubule-based kinesin motor [3], and suppression of CENP-E leads to defects characterized by an intact bipolar spindle with several chromosomes clustered close to the poles [4]. Studies combining electron microscopic analyses with siRNAmediated suppression indicated that CENP-E deficiency correlated with a failure of mono-oriented chromosomes to congress to the equator [5-6]. In addition, expression of motorless CENP-E [7-8], produced a similar failure in achieving metaphase alignment, suggesting the role of CENP-E motor activity in chromosome congression. However, these studies do not specifically and directly address the function of CENP-E motor in chromosome dynamics.

To probe the spatiotemporal dynamics of CENP-E function in mitosis, we screened a chemical library of structurally diversified compounds for lagging chromosome phenotypes and inhibition of CENP-E motility. One identified inhibitor was further modified to produce syntelin (Figure 1A). In in vitro motility assays using purified recombinant proteins, syntelin inhibited CENP-E motility in a dose-dependent manner with an $\mathrm{IC}_{50}$ value of $160 \mathrm{nM}$ (Figure 1B and Supplementary information, Figure S1A). Among an extensive list of mitotic kinesins examined, syntelin was found to be highly selective for CENP-E (Supplementary information, Figure S1B). Importantly, syntelin binds to different sites from those of GSK923295, a recently identified CENP-E ATPase inhibitor [9], as syntelin inhibits CENP-E mutants resistant to GSK923295 in a manner indistinguishable from that of wild type motor (Supplementary information, Figure S1B). Thus, we conclude that syntelin represents a novel class of CENP-E motor inhibitor.
Syntelin does not inhibit progression through $\mathrm{S}$ and G2 phases of the cell cycle but causes mitotic arrest with lagging chromosomes, a phenotype reminiscent of what was seen in CENP-E-suppressed cells [4]. As expected, inhibition of CENP-E by syntelin did not perturb bipolar spindles but produced misaligned chromosomes near the spindle poles (Figure 1C), similar to those of CENP-E siRNA-treated cells (Supplementary information, Figure $\mathrm{S} 2 \mathrm{~B})$. The kinetochore position relative to the pole is an accurate reporter for judging chromosome misalignment (Supplementary information, Figure S2B and S2C; [10]), our quantitative analysis indicated a relatively uniform distribution of kinetochores along the length of the spindle in CENP-E-inhibited and CENP-E-suppressed cells (Supplementary information, Figure S2D). Importantly, inhibition of CENP-E motor activity by syntelin resulted in a significant increase in cells bearing misaligned chromosomes $(31.7 \pm 6.8 \% ; P<0.05$; Supplementary information, Figure S2D), indicating that CENP-E motor activity is essential for faithful chromosome congression.

Our analyses of centromere geometry in CENP-Esuppressed cells validate that CENP-E activity is essential for centromere stretch (Supplementary information, Table S1). Misaligned chromosomes and decreased centromere stretch in syntelin-treated cells suggest that inhibition of CENP-E motor activity results in abnormal interactions between the kinetochores and spindle microtubules. In syntelin-treated cells, cold-stable kinetochoremicrotubule fibers were present on both aligned chromosomes and chromosomes near the pole (Supplementary information, Figure S3A). Interestingly, careful examination revealed that the kinetochores of lagging chromosome appeared to connect with spindle microtubules derived from the same pole (Supplementary information, Figure S3B; enlarged insets).

To study the precise kinetochore attachment in the absence of CENP-E motor activity, we carried out electron microscopic analysis on syntelin-treated HeLa cells. As shown in Figure 1D, spindle microtubules emanate from two opposing centrioles (asterisks) form a bipolar spindle with majority of chromosomes congressed near the spin- 


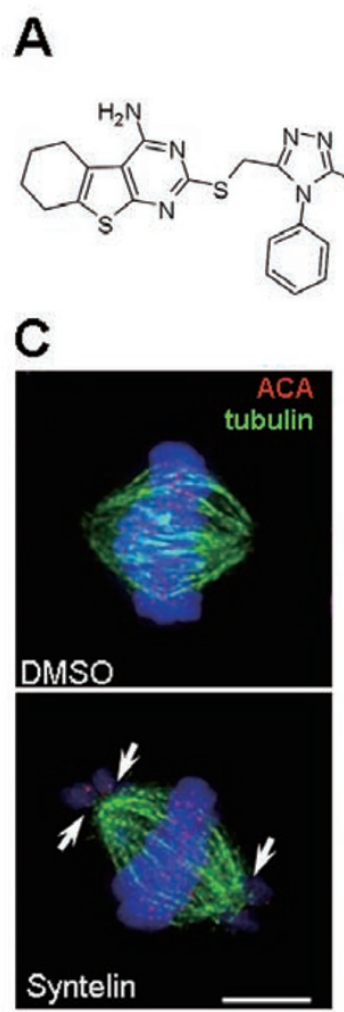

B

E
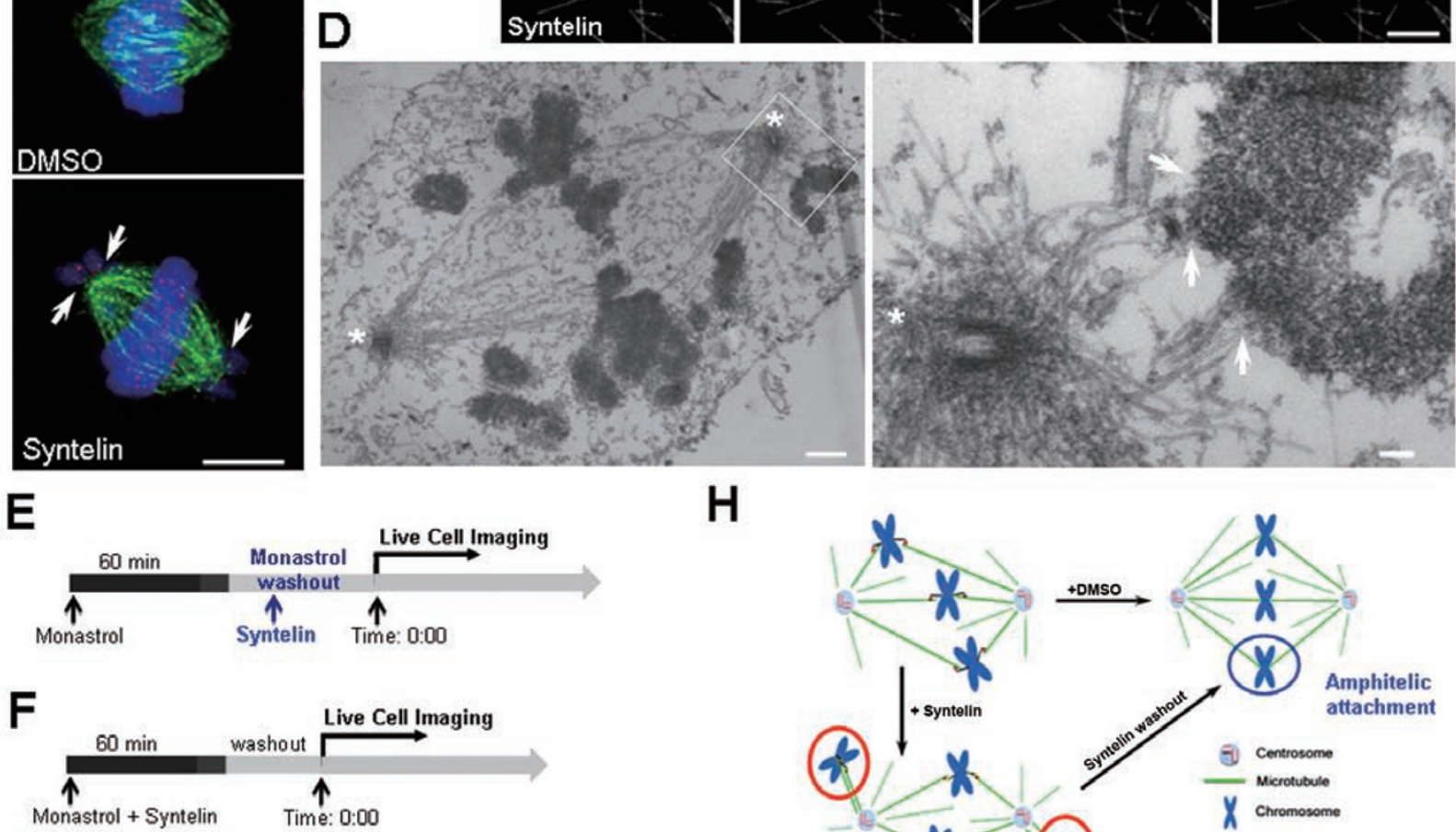

\section{G}
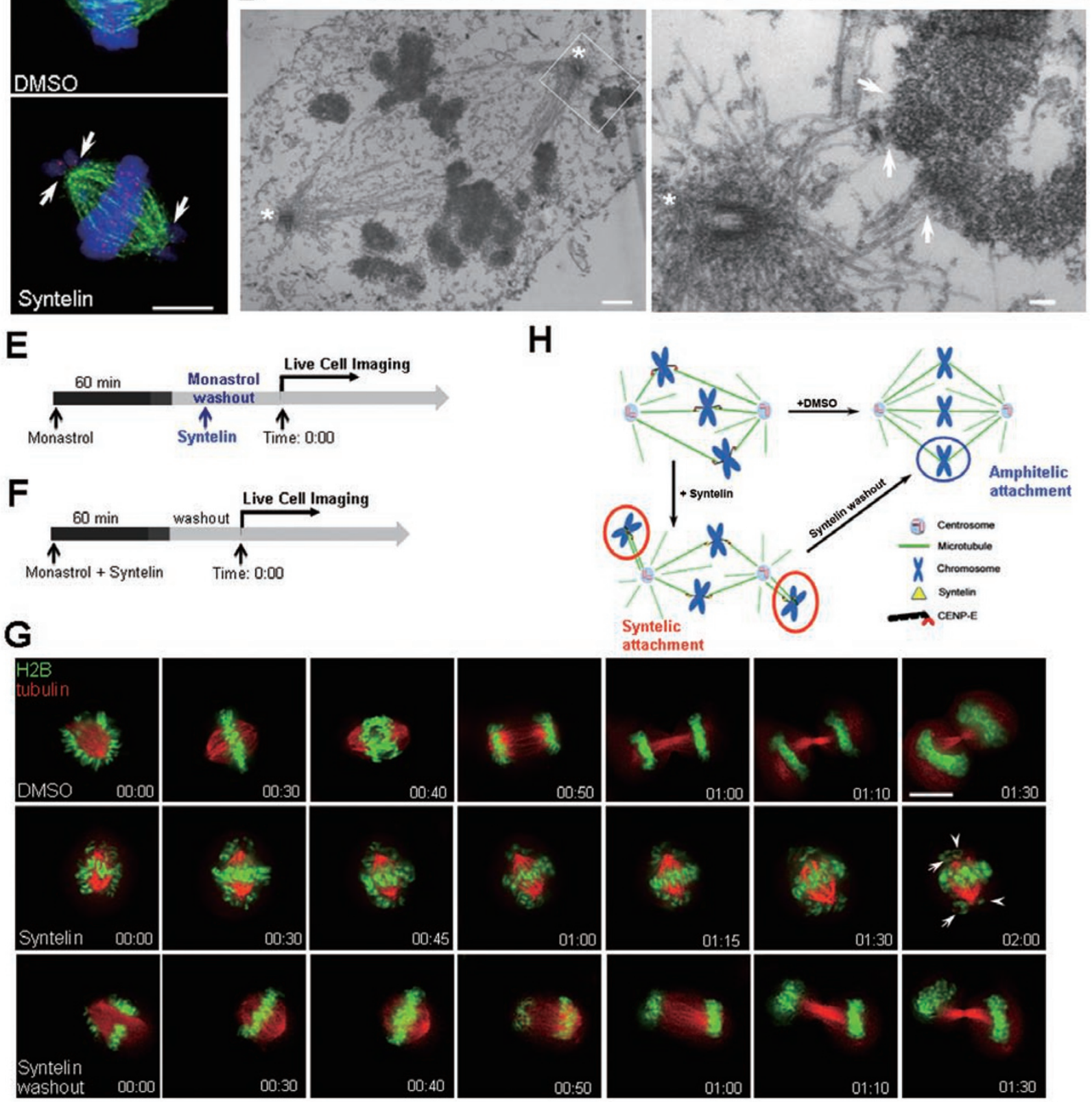

H
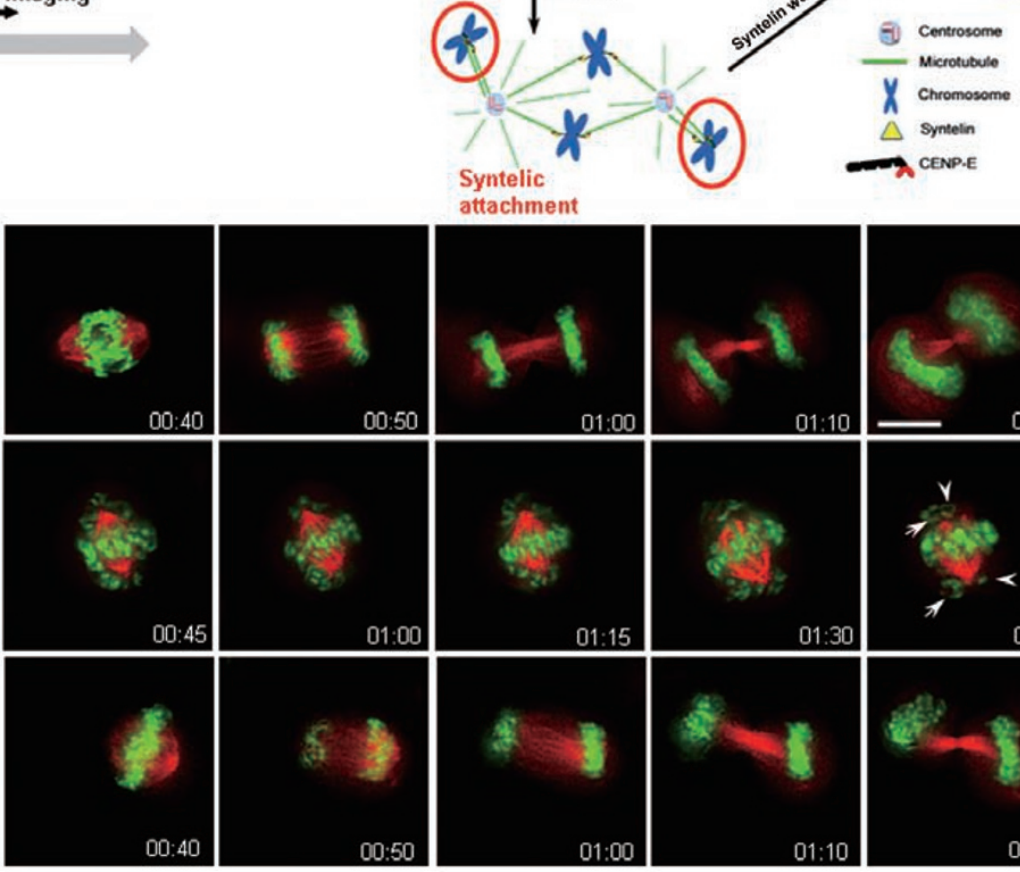

Syntelic

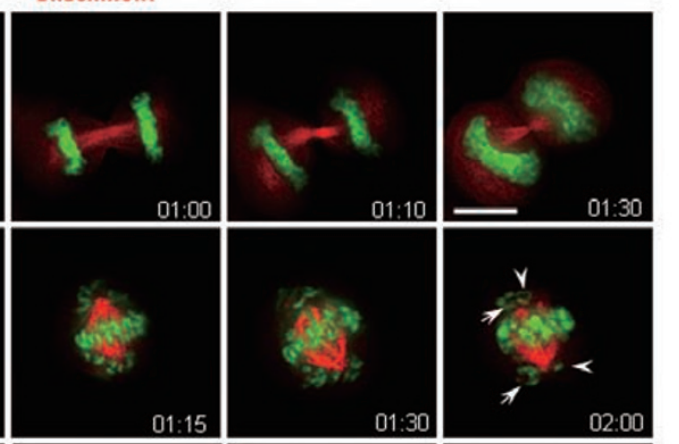


dle equator while a few chromosomes remain scattered around the poles (Figure 1D, boxed area). At a higher magnification, it was readily apparent that one chromosome near the pole displays a clear syntelic attachment in which the kinetochore connects to two sets of microtubules emanating from same centriole. Thus, CENP-E motor activity is essential for the accurate attachment of kinetochores to spindle microtubules (Supplementary information, Figure S3C).

Small molecules that modulate specific protein functions are valuable tools for dissecting complex processes in mammalian cell division. Having demonstrated the ability of syntelin to inhibit CENP-E motor function in cultured cells, we sought to test whether syntelin alters the chromosome dynamics during mitosis and whether its inhibitory effect is reversable. To this end, we adopted a protocol to synchronize cells at prometaphase and then added syntelin to probe for functional relevance of CENP-E in bipolar spindle formation and chromosome movements (illustrated in Figure 1E). We began real time imaging of prometaphase-synchronized HeLa cells expressing mCherry-tubulin and GFP-H2B soon after addition of syntelin to visualize chromosome congression and subsequent prometaphase-metaphase transition. In general, it takes an average of $41 \pm 2 \min (n=8$ cells) for HeLa cells to transit from prometaphase (mono-polar) to the anaphase onset of sister chromatid separation (Figure $1 \mathrm{G}$; top panel). However, some chromosomes still failed to align at the equator in the syntelin-treated cells even after 120 min (Figure 1G; middle panel), consistent with the essential role of CENP-E in chromosome congression [4-6].

We next examined if syntelin inhibition can be reversed using a protocol to synchronize cells at prometaphase followed by three washes to allow cells to recover from prometaphase chromosome misalignment arrest and to progress into metaphase and subsequent sister chromatid separation (Figure 1F). As predicted, syntelin removal was readily apparent as chromosomes in the released cell effectively approached metaphase alignment about 40 min after wash-out and subsequently progressed into anaphase onset (Figure 1G; bottom panel). The temporal order of metaphase-anaphase transition and telophase process in syntelin-released cells is indistinguishable from that of monastrol-released cells, demonstrating that mitotic arrest due to syntelin treatment is also rapidly reversible. Synthelin is thus a useful tool to dissect the molecular mechanisms underlying mitotic checkpoint regulation and correction of chromosome mal-orientation.

Small molecules that modulate specific protein functions are valuable experimental tools for dissecting complex processes in mammalian cells. By using a novel CENP-E motor syntelin inhibitor, we have been able to directly address, for the first time, the requirement of CENP-E in these processes. Because the phenotypes derived from CENP-E protein repression appear more extensive than those induced by syntelin, our data demonstrated the usefulness of small molecule inhibitors in dissecting the spatiotemporal dynamics of complex chromosome movements in mitosis. Indeed, kinetochore fibers form in the presence of syntelin, suggesting that CENP-E activity is not required for spindle microtubule capture, but rather accurately regulates these interactions to ensure correct bi-orientation and promote correct chromosome alignment.

CENP-E has been implicated in spindle checkpoint satisfaction $[4,7]$ as well as chromosome alignment [5]. The temporal profiles of CENP-E distribution to the kinetochore in prometaphase cells and its dissociation from aligned kinetochores at metaphase are consistent with this notion. Indeed, inhibition of CENP-E motor activity retains active spindle checkpoint judged by the localization of Mad2 to the kinetochore of lagging chromosomes near the spindle poles (Supplementary information, Figure S4). This mitotic arrest is relieved and active checkpoint silenced upon removal of CENP-E inhibition by syntelin washout. Thus, in human cells at least, CENP-E activity appears to be directly required for spindle checkpoint silencing and correct kinetochore attachment. It would be of great interest to directly address and quantify the checkpoint kinase activity on syntelic attachment and examine what happens in live chromosome congression

Figure 1 Syntelin selectively inhibits CENP-E motor activity. (A) Chemical structure of syntelin. (B) CENP-E motility and syntelin inhibition. Minus-end-marked microtubules were added with $1 \mathrm{mM}$ ATP to a flow chamber containing purified CENP-E tethered to the coverslip with an anti-histidine antibody. Gliding of microtubules was monitored by a wide-field decovolution fluorescence microscope in the presence or absence of syntelin. Selected frames from one time lapse series, spaced 30 sec apart, are presented. The average microtubule velocity of all microtubules was $5.3 \mu \mathrm{m} / \mathrm{min}$. Scale bar, $5 \mu \mathrm{m}$. (C) HeLa cells treated with $1 \mu \mathrm{M}$ syntelin for 30 min before staining for tubulin, ACA and DAPI. Misaligned chromosomes are marked by arrows. Bar: $5 \mu \mathrm{m}$. (D) Electron microscopic view of a HeLa cell treated with $1 \mu \mathrm{M}$ syntelin (poles labeled with asterisks). Bar: 1 $\mu \mathrm{m}$. Magnified view of syntelic attachments (arrows). Bar: $100 \mathrm{~nm}$. (E-F) diagram of real-time experiments for assessing the precise function of syntelin. (G) real-time imaging of HeLa cell division with syntelin and syntelin wash-out. (H) working model accounting for syntelin action in mitotic chromosome movements. 
upon the removal of syntelin and correction of syntelic attachment.

In summary, our chemical biological study of mitotic kinesins led to identification of a novel CENP-E motor inhibitor, syntelin. Chromosomes in syntelin-treated cells frequently exhibit syntelic attachment in which both sister kinetochores attached to spindle microtubules from the same pole. Syntelin is thus a useful tool to study mitotic checkpoint regulation and correction of chromosome malorientation, and contributions of CENP-E to chromosome dynamics and plasticity.

\section{Acknowledgments}

We thank members of our groups for insightful discussion during the course of this study. This work was initiated by the chemical biology grant PGX-1 from the Proteomics Research Laboratory, and supported in part by Chinese Academy of Sciences Grants (KSCX2-YW-H-10 and KSCX2-YW-R195), 973 projects (2002CB713700; 2010CB912103), National Natural Science Foundation of China (90913016), and Georgia Cancer Coalition Eminent Scholar Award.

Xia Ding ${ }^{1,2,3, *}$, Feng Yan ${ }^{1,2, *}$, Phil Yao ${ }^{2}$, Zhihong Yang ${ }^{4}$, Weihong Wan ${ }^{4}$, Xiwei Wang ${ }^{1,2}$, Jing Liu ${ }^{1}$, Xinjiao Gao ${ }^{1}$, Ariane Abrieu ${ }^{5}$, Tongge $\mathrm{Zhu}^{1,2}$, Jiancun Zhang ${ }^{1}$, Zhen Dou ${ }^{1,2}$, Xuebiao Yao ${ }^{1}$

${ }^{1}$ Anhui Key Laboratory for Cellular Dynamics and Chemical Biology, Hefei 230027, China; ${ }^{2}$ Georgia Cancer Coalition, Atlanta, GA 30310, USA; ${ }^{3}$ Beijing University of Chinese Medicine, Beijing 100026, China; ${ }^{4}$ Proteomics Research Laboratory, Beijing 100086, China; ${ }^{5}$ Universités Montpellier, 34293 Montpellier, France
*These two authors contributed equally to this work. Correspondence: Xuebiao Yao

E-mail: yaoxb@ustc.edu.cn

\section{References}

1 Cleveland DW, Mao Y, Sullivan KF. Centromeres and kinetochores: from epigenetics to mitotic checkpoint signaling. Cell 2003; 112:407-421.

2 Hyman AA, Mitchison TJ. Two different microtubule-based motor activities with opposite polarity in kinetochores. Nature 1991; 351:206-211.

3 Yen TJ, Li G, Schaar BT, Szilak I, Cleveland DW. CENP-E is a putative kinetochore motor that accumulates just before mitosis. Nature 1992; 359:536-9

4 Yao X, Abrieu A, Zheng Y, et al. CENP-E forms a link between attachment of spindle microtubules to kinetochores and the mitotic checkpoint. Nat Cell Biol 2000; 2:484-491.

5 Kapoor TM, Lampson MA, Hergert P, et al. Chromosomes can congress to the metaphase plate before biorientation. Science 2006; 311:388-391.

6 Cai S, O'Connell CB, Khodajakov A, Walczak CE. Chromosome congression in the absence of kinetochore fibres. Nat Cell Biol 2009; 11:832-838.

7 Mao Y, Desai A, Cleveland DW. Microtubule capture by CENP-E silences BubR1-dependent mitotic checkpoint signaling. J Cell Biol 2005; 170:873-880.

8 Schaar BT, Chan GK, Maddox P, Salmon ED, Yen TJ. CENP-E function at kinetochores is essential for chromosome alignment. J Cell Biol 1997; 139:1373-1382.

9 Wood KW, Lad L, Luo L, et al. Antitumor activity of an allosteric inhibitor of CENP-E. Proc Natl Acad Sci USA 2010; 107:5839-5844

$10 \mathrm{Du}$ J, Cai X, Yao J, et al. The mitotic checkpoint kinase NEK2A regulates kinetochore microtubule attachment stability. Oncogene 2008; 27:4107-4115.

(Supplementary information is linked to the online version of the paper on the Cell Research website.) 